\title{
Primary Care Research in Morecambe Bay CCG
}

\author{
Dr Simon Wetherell MA, BM, BCH, FRCGP
}

\section{INTRODUCTION}

Morecambe Bay Clinical Commissioning Group (CCG) is one of the leading areas for primary care research in the North West. For several years the former Lancashire North CCG has been the highest or second highest recruiter of patients for National Institute for Health Research (NIHR) studies in the North West Coast Clinical Research Network (CRN) area (covering South Cumbria to mid Cheshire). Last year we recruited 931 patients for NIHR studies in primary care. There are several practices in South Cumbria also doing excellent work for the NIHR, but I am only just now finding out about the details of these.

The North West Coast CRN is based in Liverpool, with a satellite site in Preston Business Centre. We work closely with the CRN team, and they are very supportive of the initiatives that we have been working on locally. The CRN pays for a team of research nurses based in several practices throughout Morecambe Bay, and their job is to recruit patients for studies which are on the NIHR "Portfolio". My simplistic understanding of the situation is that the CRN obtains funding from a central pot, based largely around how many patients are recruited to these NIHR studies. They, therefore, have an incentive to support areas where there is good recruitment, and they are using their funds to assist this process through spending on practice based research nurses. As we have shown good recruitment levels locally over the years, we have been favoured with a high level of investment by the CRN.

In the former Lancashire North CCG, Queen Square Medical Practice was the first to have a CRN supported research nurse, starting in 2010. We have now grown our Queen Square practice team to three research nurses. Our lead research nurse, Nicky Harding, also has a role co-ordinating the work of the further three research nurses who now cover all the other practices in Lancaster, Morecambe and Carnforth. I meet with the Queen Square nurses on at least a weekly basis and with the other local nurses periodically. The local research nurses also meet with other CRN funded nurses at regional meetings, and I keep in close contact with managers and other clinical leads at the CRN. We are starting to make more connections with research nurses and practices in South Cumbria, and we are hoping to appoint a new nurse with a remit to enhance this work shortly.

\section{RESEARCH STUDIES}

There are basically two types of NIHR Portfolio research project that we get involved with - academic and commercial. The majority are academic studies, and they vary considerably in their complexity. The simplest studies involve mail outs of questionnaires to specific patient groups, which demands little other than the ability to generate adequate searches. At the other end of the spectrum are medication studies such as Barak-D, in which patients with eGFRs between 30 and $50 \mathrm{ml} / \mathrm{min}$ are randomly allocated to spironolactone or no treatment to look at progression of chronic kidney disease (CKD) and cardiovascular outcomes. The eligibility criteria are complex, there are numerous follow-ups, and (from personal experience) there are multiple points where difficulties arise.
At Queen Square we only take on academic studies that all members of the research team agree to be clinically important and practically doable. We are involved in some studies that I and my colleagues consider to be clinically extremely relevant to primary care practice. We are just coming to the end of recruiting for a study called "AllHeart" which randomly allocates patients (without any previous episodes of gout) who have a history of ischaemic heart disease to either allopurinol or no treatment, as there is theoretical but unproven clinical benefit with this drug. We are also involved with a study comparing sertraline to placebo in a double blind trial for patients with mild depression (the "PANDA" study). Very topically, we are recruiting patients who have been intolerant of statins due to myalgia to a double blind study called "Statinwise", in which they will receive statins or placebo at different times while recording any symptoms. We are nearing the end of recruiting to "Candid", which looks at the symptoms and signs of patients presenting with lower gastrointestinal or lung problems, and they are then followed up long term for the development of cancer.

It is always gratifying to see studies in which have had a role being published in high impact journals. A study looking at oral steroid use in lower respiratory tract infections in adults without asthma is just being published in JAMA. The "DUTY" study, looking at assessing pyrexial children for urinary tract infection (UTI) was recently published in the RCGP journal and the "CPSS" study, looking for familial hypercholesterolaemia, was recently published in the New England Medical Journal.

Commercial studies are often more complex. In our experience they are often very clearly and tightly designed, but the criteria tend to be extremely strict, and the inputs required from the practice are significant. This is not always the case, but if the study is looking at a new drug, then it is likely to be complicated. We are currently involved in a study looking at a new treatment for diarrhoea, which is a placebo controlled study. There are occasionally less complex commercial studies such as one that we are about commence, which is surveillance for complications in patients receiving flu vaccination.

\section{UNIVERSITY LINKS}

We have started working closely with Lancaster University. The first way that we work with them is demonstrated by our links with the Spectrum Centre. They have contacted us about studies that they are running for patients with bipolar disorder. These studies have full NHS approval. We send out the literature about the study to the appropriate patients (after screening out patients who should not be contacted) - the Spectrum Centre only gets to know about any details relating to individual patients if they respond to the invitation to be involved. The University then does the rest without our involvement. We have worked on similar lines with the Centre for Ageing Research and the End of Life Observatory.

We get more closely involved with some studies organised by University academics. We are about to start a study looking at rapid eye movements in patients with cognitive impairment, which we will be recruiting for at the surgery. We were directly involved in helping set up 
and run a study looking for Influenza $\mathrm{C}$ in patients with and without upper respiratory symptoms, which has resulted in several publications by Derek Gatherer, who is a University virologist. Three Morecambe Bay practices are just about to start a project with a University researcher looking at the "fit note" process.

However, what I most wish to achieve is research generated by ideas from local primary care clinicians. I work with Steve Milan from the University. His role is to connect interested clinicians with appropriate academics. Andy Knox is working on several research projects around the theme of improving lifestyle to improve health. There are several other local clinicians involved with University academics, looking at working up a research proposal for funding. Because we recruited more than 500 patients to NIHR Portfolio academic studies locally last year, we have been allocated 220,000 Research Capability Funding by the NIHR. The directions for the use of this funding state that it must be used to develop and promote research. In my communications with the CRN management, they have strongly supported using this money to help free up time for primary care clinicians to work with University academics in setting up proposals for funding of research projects. There is still some funding for use this year, and I am very keen to hear from anyone from primary care with a research idea that they would like to pursue, however vague it might be at this present time. There is no guarantee that this funding will be available next year, so do not hesitate.

Other areas have helped promote high quality GP recruitment by clinical fellowships between the CCG and local university. There is a new post that is soon to be created here to allow a local GP to spend time doing a PHD alongside some clinical practice. Elsewhere in the country registrars have done an MSc in Health Research alongside a lengthened GP registrar clinical training - there is local interest in this, but alas, at present, local financial pressures are preventing this aspiration from being fulfilled.

\section{SUCCESSES}

We are doing really well with our recruitment to NIHR studies, and hence the awarding of the Research Capability Funding for last year. Lancashire North CCG research team were finalists in the Health Service Journal research team of the year category, and we are in the final of the Nursing Times research team of the year awards, the results of which will be announced in November - on both occasions we have been the only research team from primary care in the finals. We also won the North West Coast research team of the year awards earlier this year, covering primary, secondary and community care. Queen Square won the national RCGP/NIHR practice team award for research in 2016.

The reasons for this local success are, as ever with such matters, multifactorial. We have a superb team of research nurses, along with supportive management at the CRN. The local practices have embraced research; Lauren Butler (from the Lancashire North GP Federation) in particular helped to break down barriers, and get practices involved with research. At my own practice (and I am sure many others) all staff, including especially the receptionists, have become engaged with the research processes - they are aware of the projects, and help highlight them to relevant patients. We have a group of interested GPs who are keen to increase the research at their own practices. Our patients have become aware of the amount of research being done in primary care, and rarely do we encounter any surprise from them when the concept of being involved in a research project is broached. We seem to have moved into a bit of a virtuous circle, whereby things go well and promote greater research activity - I hope this will continue.

\section{THE FUTURE}

The future, as always, holds threats as well as exciting prospects and opportunities. Our research nurse team should be expanding soon, with increased connections between nurses in North Lancashire and South Cumbria. I hope we can encourage new practices to join our network it does make some money for the practice (though I cannot promise that this will be in large amounts); but, more importantly, involvement in research is not only stimulating for the clinician, it is good for clinical patient care at the practice and there is good evidence to support this. There are opportunities to develop locally grown research projects, and I hope that we will soon establish some contacts with researchers at the University of Cumbria. Margaret Cooper, head of research at UHMB, may help us work on some joint projects between primary and secondary care.

The main threat that I can see is the continuing availability of good primary care research projects for us to get involved with. One of the main roles of the CRN is to entice researchers to bring their studies to this area. When the researchers look at the map see where Morecambe Bay is situated, they sometimes run away. We need a strong research recruitment record to show them that we can and will deliver. If we continue to do that, then we should be able to attract studies to this far flung corner of the country. The other thing we can do to futureproof ourselves is to encourage locally grown research projects, in conjunction with the universities. These need to be on the NIHR Portfolio for us to involve the research nurses and to gain the recruitment accrual that counts towards CRN funding, and this is a bit of an extra hurdle. We have one of the top universities in the country on our doorstep, and it benefits both parties to work more closely - as the medical school "matures" I am sure we will see increased interest in medical research being generated locally, and we need to be ready and available to be part of this process in order to access the benefits.

\section{CONCLUSION}

Primary care research in the Morecambe Bay area is currently going very well, with large numbers of patient recruits to NIHR sturdies. We have plans to expand further, and interested practices can contact me or Nicky Harding at Queen Square Medical Practice in Lancaster. We need to build our own local research projects, in conjunction with the local universities. Anyone with an idea for a primary care research project is very welcome to make contact to discuss this further.

All of the authors are funded/part funded by the National Institute for Health Research, Collaboration for Leadership in Applied Health Research and Care North West Coast (NIHR CLAHRC NWC). The views expressed are those of the author(s) and not necessarily those of the NHS, the NIHR or the Department of Health.

Correspondence to: Simon.Wetherell@gp-p81013.nhs.uk 